Revisión bibliográfica

Volumen 32(3): 1034-1046. Septiembre-diciembre, 2021 e-ISSN 2215-3608, doi:10.15517/am.v32i3.43610 https://revistas.ucr.ac.cr/index.php/agromeso/index

\title{
Bananos (Musa AAA): Importancia, producción y comercio en tiempos de Covid-191
}

\section{Bananas (Musa AAA): Importance, production and trade in Covid-19 times}

\author{
Gustavo E. Martínez-Solórzano ${ }^{2}$, Juan C.Rey-Brina ${ }^{2}$
}

1 Recepción: 31 de agosto, 2020. Aceptación: 25 de febrero, 2021. Este trabajo formó parte de resultados de actividades de investigación relacionadas con la fitosanidad en sistemas de producción de Musáceas comestibles en Venezuela, ejecutado por el Centro Nacional de Investigaciones Agrícolas (CENIAP), del Instituto Nacional de Investigaciones Agrícolas, Venezuela.

2 Instituto Nacional Investigaciones Agrícolas (INIA), Centro Nacional Investigaciones Agropecuarias (CENIAP). Maracay, Venezuela. martinezgve@yahoo.es (autor para la correspondencia, https://orcid.org/0000-0003-2599-1712); jcrey67@ gmail.com (https://orcid.org/00000001-7271-3606).

\section{Resumen}

Introducción. En la actualidad los bananos son las frutas más consumidas en el mundo, considerados como cultivos estratégicos en la seguridad alimentaria de muchos países. Sin embargo, ante la eventual pandemia de COVID-19, se generan efectos colaterales que afectan su actividad económica a nivel mundial. Objetivo. Señalar los rasgos más significativos de los bananos en relación con su importancia en el ámbito económico y productivo ante las condiciones generadas por la pandemia COVID-19, así como las amenazas fitosanitarias actuales como factores limitantes para su producción. Desarrollo. El banano es la base de la economía y dieta de muchos países, ocupa un importante lugar en la producción y comercio internacional, al ser la fruta más exportada, representado en su mayoría por triploides AAA Cavendish. Su consumo aporta vitaminas, minerales y otros elementos con propiedades medicinales como la lectina, que puede actuar como agente antiviral de amplio espectro. No obstante, en la actualidad se ha generado disrupciones en el suministro del mismo, lo que afecta todos los elementos de la cadena productiva, producto de la situación originada por la COVID-19. Conclusiones. La pandemia del coronavirus ha generado efectos colaterales que involucra diversas áreas como la producción y comercio de bananos y se encuentran asociados al aislamiento social y restricción de movilidad. Sin embargo, se considera que el banano seguirá siendo la fruta más importante dentro de ese rubro, destacándose entre los cultivos de más rápido crecimiento en la agricultura, a pesar de las condiciones originadas por la COVID-19 y la existencia de muchas incertidumbres causadas por enfermedades emergentes y perturbaciones climáticas, que pueden afectar su entorno económico y productivo.

Palabras claves: Musaceae, cultivos, suministro de alimentos, pandemias, enfermedades de las plantas.

\begin{abstract}
Introduction. Bananas are currently the most consumed fruits in the world, and considered strategic crops in food security in many countries. However, in the event of a COVID-19 pandemic, side effects are generated that affect its economic activity worldwide. Objective. To point out the most significant features of bananas in relation to their economic and productive importance in the face of the conditions generated by the COVID-19 pandemic, as well as
\end{abstract}


the current phytosanitary threats as limiting factors for their production. Development. Bananas are the basis of the economy and diet of many countries, occupying an important place in international production and trade, being the most exported fruit, mostly represented by triploid AAA subgroup Cavendish. Its consumption provides vitamins, minerals, and other elements with medicinal properties, such as lectin that acts as a broad-spectrum antiviral agent. However, at present there have been disruptions in its supply, which affects all elements of the production chain, as a result of the situation caused by COVID-19. Conclusions. The coronavirus pandemic has generated collateral effects that involve various areas such as the production and trade of bananas, and are associated with social isolation and restricted mobility. However, it is considered that bananas will continue to be the most important fruit within this sector, standing out among the fastest growing crops in agriculture, despite the conditions caused by COVID-19 and the existence of many uncertainties caused by emerging diseases and climatic disturbances, which can affect the economic and productive environment.

Keywords: Musaceae, crops, food supply, pandemics, plant diseases.

\section{Introducción}

En la actualidad, los bananos son el primer producto globalizado del mundo moderno y siguen siendo las frutas más exportadas, las más consumidas y uno de los principales productos que conforman el movimiento diario en el mercado internacional (Crawford \& Kueffner, 2020; Food and Agriculture Organization [FAO], 2020a). Aun cuando los datos referentes a su producción, consumo y comercio, pueden subestimarse por el carácter extensivo del cultivo que incluye pequeñas parcelas familiares, la información disponible refleja su importancia en la oferta global, que ha aumentado en las últimas décadas (FAO, 2020a). Los bananos poseen cualidades alimenticias y medicinales que los diferencian del resto de las frutas, son considerados como cultivos estratégicos en la seguridad alimentaria de muchos países (Martínez et al., 2020). No obstante, ante la eventual situación generada por el coronavirus (SARS Cov-2), uno de los riesgos que todos los países han contemplado tras la aplicación de las medidas sanitarias para enfrentar su propagación, han sido las posibles disrupciones al adecuado funcionamiento del suministro de alimentos, donde se incluye el banano (FAO, 2020d; 2020e).

Este trabajo tuvo como objetivo señalar los rasgos más significativos de los bananos en relación con su importancia en el ámbito económico y productivo ante las condiciones generadas por la pandemia COVID-19, así como las amenazas fitosanitarias actuales como factores limitantes para su producción.

\section{Origen y distribución}

El banano es una de las frutas más antiguas cultivadas y es considerado como un cultivo muy evolucionado (Rekha et al., 2001). Estudios arqueológicos revelan hallazgos de las primeras plantas en el sudeste asiático en Papúa Nueva Guinea, donde se presume fue domesticado por agricultores hace 7000 a 10000 años (Li et al., 2013; Organización de las Naciones Unida [ONU], 2016; Soto, 1992).

Se señalan a Musa acuminata y Musa balbisiana como ancestros de los bananos actuales, que aportan los genomas A y B, respectivamente, también se indican otros ancestros como M. schizocarpa (sección Eumusa) y M. textilis (sección Callimusa) (donantes del genoma S y T, respectivamente), involucrados en los cruces o hibridación para la formación de los nuevos bananos (Heslop-Harrison \& Schwarzacher, 2007; Li et al., 2013; Perrier et al., 2011; Rekha et al., 2001). Aún cuando estos últimos no muestran una diversificación morfológica evidente, en Papua Nueva Guinea, se ha logrado identificar cultivares producto de la hibridación con M. schizocarpa, mientras que en 
Filipinas, como resultado de una hibridación entre M. balbisiana y M. textilis (Li et al., 2013; Rekha et al., 2001). La ocurrencia de los genomas de M. shizocarpa y M. textilis entre el complemento cromosómico de cultivares de Musa, revelan la complejidad de la estructura genómica de los cultivares actuales de banano y su evolución, lo que queda en evidencia a través de estudios genéticos recientes, que las bananas cultivadas se originaron en el género Musa a través de complejas vías de geodomesticación (Li et al., 2013; Perrier et al., 2011; Rekha et al., 2001).

De la especie Musa acuminata, se han identificado al menos nueve subespecies (banksii, burmannica, burmannicoides, errans, malaccensis, microcarpa, siamea, truncata y zebrina), mientras que aún cuando no se han designado categorías de subespecies en M. balbisiana, también exhibe una amplia variación en los caracteres morfológicos (Li et al., 2013; Rekha et al., 2001).

Los bananos actuales difieren de sus parentales silvestres por no poseer semillas y ser partenocárpicos ( $\sin$ desarrollo de semillas o polinización y fertilización) y aún cuando se reproducen a través de la propagación vegetativa, exhiben alto nivel de diversificación morfológica en tamaño, forma y color del fruto (Heslop-Harrison \& Schwarzacher, 2007; Li et al., 2013).

Los cultivares diploides AA actuales provienen de M. acuminata y basados en análisis filogenéticos, se indica que fueron domesticados en las islas del sudeste asiático (ISEA), a través de múltiples hibridaciones intraespecíficas entre diferentes subespecies de M. acuminata, donde las subespecies malaccensis, banksii y errans, contribuyeron con la mayoría de estos cultivares, lo cual sugiere múltiples orígenes maternos, que pueden ser una característica general de estos cultivares AA (Heslop-Harrison \& Schwarzacher, 2007; Li et al., 2013; Perrier et al., 2011). Mientras que los bananos AAA cultivados se derivan de hibridaciones entre cultivares AA, donde la triploidización pudo ocurrir por separado en varias regiones geográficas a través de múltiples hibridaciones entre diferentes cultivares AA y su posterior diseminación a otras regiones del mundo (Heslop-Harrison \& Schwarzacher, 2007; Li et al., 2013; Perrier et al., 2011). Así, los cultivares Gros Michel (AAA) y Grande Naine (AAA), tienen un ancestro diploide común (Li et al., 2013).

La alta diversidad de nucleótidos en el banano cultivado, implica que pudo existir una población que no experimentó ningún cuello de botella genético severo durante el proceso de domesticación. Sin embargo, se observaron altos grados de divergencia fenotípica entre diferentes cultivares, lo que sugiere que la selección artificial pudo haber actuado sobre rasgos morfológicos, de tal manera que disminuyera la diversidad genética de los bananos domesticados (Li et al., 2013).

En el proceso de domesticación, las migraciones humanas e intercambios de material vegetal, hicieron posible situar al banano en contextos ecológicos diferentes, donde los agricultores aprovecharon las mutaciones naturales y multiplicación vegetativa que originaron la diversidad genética actual (ONU, 2016). A través de la historia se evidencian tres eventos relacionados con su distribución: 1) desde el Sudeste Asiático y Papúa Nueva Guinea hasta la península del Indostán y el Pacífico, mediante las migraciones poblacionales, 2) en el siglo XV los comerciantes árabes y persas lo llevaron desde el Sudeste Asiático hasta Oriente y luego a África y Europa y 3) hacia las islas del Caribe y el Nuevo Mundo por exploradores, colonizadores y misioneros europeos (ONU, 2016; Perrier et al., 2011).

El banano no se conoció en el Mediterráneo como cultivo hasta el año 650. Su introducción a Egipto y África fue gracias a antiguos mercaderes de oriente y/o árabes durante sus expediciones (ONU, 2016; Soto, 1992). Su introducción a Santo Domingo (República Dominicana), fue a través de algunas plantas procedentes de las Islas Canarias en el año 1516, identificándose los primeros clones en el Nuevo Mundo como "Silk Fig" y "French Plantain" (Soto, 1992).

No se conoce con exactitud en qué fecha se estableció el clon "Gros Michel" como explotación comercial en el Caribe y Centroamérica, pero se presume que fue introducido en 1836 y las primeras siembras comerciales se llevaron a cabo en Jamaica y Panamá antes del año 1866, desarrollándose a partir de 1870 su explotación a gran escala (ONU, 2016; Soto, 1992) y domina el mercado internacional hasta la década de los años cincuenta, cuando fue sustituido por clones del subgrupo Cavendish, por el efecto devastador de la raza 1 del hongo Fusarium oxysporum sp. cubense (Foc), causante de la enfermedad de Marchitez por Fusarium. 


\section{Importancia}

En muchos países, esta fruta es importante fuente de crecimiento económico e ingresos para muchas zonas rurales, al generar empleos y divisas, siendo representada en su gran mayoría por triploides $A A A$, subgrupo Cavendish, mientras que en otros países presenta mayor importancia comercial que la industria petrolera, tal como en Ecuador, donde después del colapso de los precios del crudo en el año 2019, el mercado del banano adquirió mayor importancia para la economía (FAO, 2020e).

Entre las cualidades del banano, se puede indicar que $100 \mathrm{~g}$ de pulpa de fruta madura contiene $70 \mathrm{~g}$ de agua, 0,3 $\mathrm{g}$ de grasa, $27 \mathrm{~g}$ de carbohidratos, $1,2 \mathrm{~g}$ de proteínas, $0,5 \mathrm{~g}$ de fibra y puede producir aproximadamente 90 calorías. Además, posee magnesio (30-35 mg $100 \mathrm{~g} \mathrm{~g}^{-1}$ ), potasio (385-500 mg $100 \mathrm{~g} \mathrm{~g}^{-1}$ ), fósforo (22-30 mg $\left.100 \mathrm{~g}^{-1}\right)$, calcio (3-8 mg $\left.100 \mathrm{~g}^{-1}\right)$, hierro $\left(0,42-0.6 \mathrm{mg} 100 \mathrm{~g}^{-1}\right)$ y zinc $\left(0,18 \mathrm{mg} 100 \mathrm{~g}^{-1}\right)$. Es rico en vitamina $\mathrm{C}(10-20 \mathrm{mg}$ $\left.100 \mathrm{~g}^{-1}\right)$, riboflavina $\left(0,04-0,07 \mathrm{mg} 100 \mathrm{~g}^{-1}\right)$, tiamina $\left(0,04-0,08 \mathrm{mg} 100 \mathrm{~g} \mathrm{~g}^{-1}\right)$, ácido pantotenina $\left(0,26 \mathrm{mg} 100 \mathrm{~g}^{-1}\right) \mathrm{y}$ piridoxina $\left(0,51 \mathrm{mg} 100 \mathrm{~g}^{-1}\right)$ (Hapsari \& Lestari, 2016). Posee además varios antioxidantes como la galocatequina y la dopamina (Kanazawa \& Sakakibara 2000; Someya et al., 2002) y bajo contenido de colesterol y sal (Anyasi et al., 2013; Zomo et al., 2014).

Los altos valores de nutrientes del banano, pueden satisfacer las necesidades diarias de personas de cualquier edad, proporcionando hasta $23 \%$ de potasio, que contribuye a disminuir la presión arterial y el riesgo de accidentes cerebrovasculares (Blasco \& Gómez, 2014; Hernández \& Vit, 2009); 41 \% de vitamina B6 que ayuda a disminuir la depresión y síndrome pre-menstrual, debido que está compuesta por piridoxal, piridoxina y piridoxamina, que tienen un papel atenuante en el metabolismo de varios neurotransmisores (serotonina, norepinefrina, sistema colinergénico y ácido $\gamma$-aminobutírico) (Blasco \& Gómez, 2014). Aporta vitaminas A, C y D, brinda beneficios para los huesos y músculos del cuerpo humano, contribuye en conjunto al buen funcionamiento del metabolismo energético y del sistema nervioso y a mantener un buen tránsito digestivo (Blasco \& Gómez, 2014; Hapsari \& Lestari, 2016).

El consumo del banano mejora la asimilación de calcio, nitrógeno y fósforo en el cuerpo (ayuda a la reconstrucción de tejidos), además de contribuir a combatir desórdenes intestinales como úlceras, siendo uno de los pocos frutos que pueden consumir estos pacientes sin generarles algún problema, además de ser excelente fuente de potasio (Blasco \& Gómez, 2014; Kumar et al., 2012). Son frutas climatéricas, responsables del aumento del nivel de carbohidratos asociados al almidón cuando la fruta es ingerida madura (Anyasi et al., 2013). En estado inmaduro, presentan alta concentración de almidón (70 \% en comparación con los maduros), el cual se degrada a una pequeña porción de monosacáridos, mientras que el resto del almidón se degrada a sacarosa (Blasco \& Gómez, 2014).

Los bananos poseen lectina, que es una proteína de carbohidratos que puede intervenir en muchos procesos biológicos y funciona como agente antiviral de amplio espectro (inductor de Antígeno IgG4), que puede actuar contra los virus de la hepatitis C (VHC) y la influenza, y contra el desarrollo o progresión del cáncer del páncreas y contra algunas bacterias y protozoarios (Gavrovic et al., 2008; Swanson \& Markovitz, 2015). Aún cuando la cantidad de lectina en la fruta es baja $(0,04 \mathrm{mg})$ y depende de su etapa de maduración, su síntesis y producción es posible, pudiendo generarse hasta $50 \mathrm{mg} \mathrm{l}^{-1}$ de cultivo celular, el cual presenta ventajas sobre las moléculas naturales con respecto a su pureza y homogeneidad (Gavrovic et al., 2008).

Otros subproductos o restos vegetales del banano también pueden poseer algunas propiedades. Las cáscaras representan el $30 \%$ del peso de la fruta (200 t/año de subproducto en el procesamiento en Tailandia). Son fuentes naturales de antioxidantes, proteínas y fitoquímicos que actúan en la eliminación de radicales libres (Arawande \& Komolafe, 2010; Kanazawa \& Sakakibara, 2000; Someya et al., 2002), también podrían representar insumos para la elaboración de alimentos funcionales que ayudan a prevenir enfermedades del corazón, cáncer y otras (Blasco \& Gómez, 2014; Kanazawa \& Sakakibara, 2000; Kumar et al., 2012; Someya et al., 2002; Ugye \& Nyiaatagher, 2009; Wachirasiri et al., 2009). 
Se ha logrado identificar en el banano la galocatequina en concentraciones de $160 \mathrm{mg} 100 \mathrm{~g}^{-1}$ en base seca, la cual se relaciona con la capacidad antioxidante de la cáscara, además de otros compuestos como antocianinas (delfinidina y cianidina), catecolaminas, carotenoides ( $\beta$-caroteno catalogado como principal precursor de la vitamina A y $\alpha$-caroteno), diferentes xantofilas, esteroles y triterpenos, como el $\beta$-sitoesterol, stigmasterol, campesterol, cicloeucalenol, cicloartenol y cicloartanol 24-metileno (Blasco \& Gómez, 2014; Kanazawa \& Sakakibara, 2000; Someya et al., 2002).

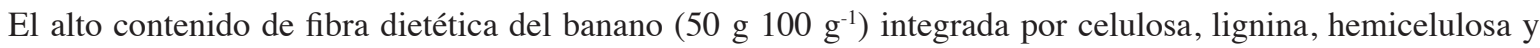
pectina (Emaga et al., 2007; Wachirasiri et al., 2009), contribuye a disminuir patologías como cáncer de colon y la arterosclerosis, reducción del colesterol en la sangre y desarrollo de la flora intestinal, mejora el control de la glucemia y la sensibilidad a la insulina en individuos diabéticos (Blasco \& Gómez, 2014). Pueden además presentar valores altos de aminoácidos esenciales como leucina, valina, fenilalanina y treonina, que contribuyen a la regulación del metabolismo (Blasco \& Gómez, 2014; Emaga et al., 2007). Presentan ácidos grasos poliinsaturados, ácidos grasos esenciales como el ácido linoleico y ácido a-linolénico, cuyo consumo es muy importante durante el embarazo y la lactancia, para prevenir el cáncer, enfermedades oculares asociadas a la edad y el Alzheimer, así como enfermedades cardiovasculares (Blasco \& Gómez, 2014). Además posee una fuente potencial de sustancias antioxidantes y propiedades antimicrobianas (Saif \& Hashinada, 2005).

Con respecto al resto de la planta de banano, la raíz puede ser utilizada para tratar enfermedades digestivas, las flores presentan alto contenido de fibra (lignina, celulosa y hemicelulosa) y minerales (potasio, sodio y calcio), con alta capacidad antioxidante y pueden ser utilizadas para tratar estrés oxidativo, disentería, úlceras y bronquitis, además de ser excelente alimento para los diabéticos una vez cocidas (Blasco \& Gómez, 2014; China et al., 2011).

Las hojas de la planta de banano pueden ser utilizadas como compresa fría para quemaduras y/o heridas (Kumar et al., 2012). El pseudotallo y rizoma tienen capacidad antioxidante y podrían ser utilizados en la elaboración de bebidas funcionales (Saravanan \& Aradhya, 2011). La savia tiene propiedades astringentes y se puede utilizar para tratar amplia variedad de enfermedades como lepra, fiebre, trastornos digestivos, hemorragia, epilepsia, hemorroides y picaduras de insectos (Blasco \& Gómez, 2014).

\section{Producción}

Entre las frutas tropicales, el banano ha sido considerado como el principal cultivo con respecto al volumen producido. Entre los años 2007 y 2018, se observó en la región de América Latina y el Caribe (LAC), incremento en la mayor parte de los periodos evaluados con el mayor y menor valor entre 2014-2015 y 2017-2018, respectivamente (Figura 1) (FAO, 2020b).

A pesar que el comportamiento del banano en el ámbito de producción global es más lento que el resto de las frutas, debido a que su demanda es impulsada y depende del crecimiento de la población, pudiendo estar saturada en la mayoría de las regiones, la Organización de las Naciones Unidas para la Alimentación y la Agricultura (FAO, 2020a) estima que para el año 2028 podría representar aproximadamente el $53 \%$ del total de la producción mundial de frutas tropicales, donde el continente asiático debería de mantener su importante participación en la producción con el mayor aporte (54\%), mientras que la región LAC (Ecuador, Brasil, Guatemala, Colombia, Costa Rica y México) podría alcanzar los 34 millones de toneladas, estimulada por la demanda de importaciones de clientes clave de los mercados desarrollados.

Ante la actual pandemia de COVID-19, se generan efectos colaterales que afectan la actividad económica a nivel mundial. En términos generales, esta pandemia repercutirá en incremento del hambre y la pobreza en diversos países, debido a que afectará la producción de alimentos en los distintos niveles y la magnitud del impacto en 


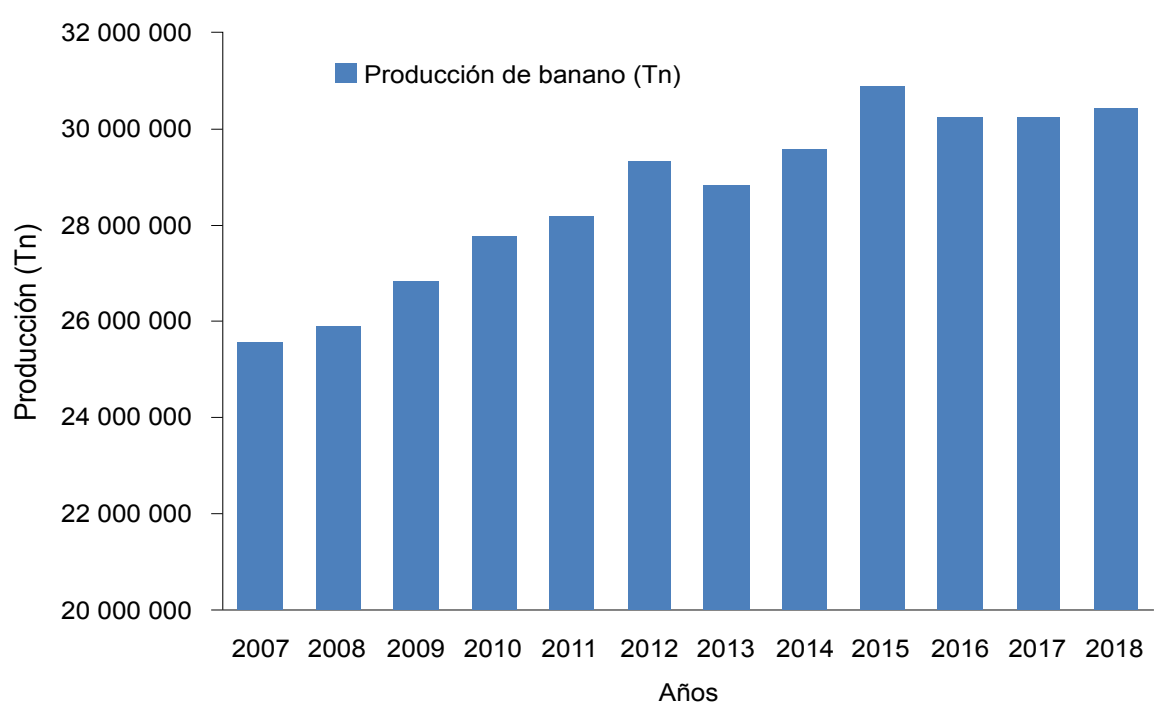

Figura 1. Producción de banano en Latinoamérica y Caribe entre los años 2007 y 2018. Fuente: FAO (2020b).

Figure 1. Banana production in Latin America and the Caribbean between 2007 and 2008. Source: FAO (2020b).

cada país, dependerá de las estrategias políticas implementadas para abordar en forma simultánea las dimensiones sanitarias y socioeconómicas de la crisis (FAO, 2020e; FAO, 2020f).

Aún cuando América Latina y el Caribe producen y tiene reservas suficientes para alimentar de forma adecuada a sus habitantes en los próximos meses, el principal riesgo en corto plazo es no poder garantizar el acceso a los alimentos de la población que está cumpliendo con las medidas de seguridad sanitaria para evitar la propagación del virus, que en muchos casos ha perdido su principal fuente de ingresos (FAO, 2020e).

En el caso específico del banano, los sistemas operativos de producción son afectados en gran medida por las medidas de restricción de movilidad o de aislamiento social que pueden crear posibles disrupciones al adecuado funcionamiento para la producción. La prioridad inicial se ha focalizado en mantener la capacidad de producción y, para ello, se deben superar barreras limitantes como disponibilidad de insumos, capital y mano de obra (FAO, 2020e). La evidencia muestra que en algunos países el sector agrícola pareciera estar cumpliendo un rol de contrarrestar, al menos en forma parcial, los impactos económicos negativos de la COVID-19 y muy probable que ayude a proteger los ingresos de los pequeños agricultores (FAO, 2020f). En muchos casos los niveles de producción han presentado índices negativos, debido a la presencia de las barreras limitantes, mientras que otros, como el caso de Camboya, han logrado mantenerse (FAO, 2020f).

\section{Consumo y comercio (exportaciones e importaciones)}

En términos de consumo, en condiciones originadas por COVID-19, se pueden generar desabastecimiento en algunos casos y/o regiones, que a su vez estará en función de aspectos inherentes a la oferta, que será una repercusión de las exportaciones, importaciones y producción en cada país. Por otro lado, por la condición de 
distanciamiento social, aislamientos y el temor de salir de casa ante la presencia del coronavirus, tanto los niveles y/o patrón de consumo pueden ser afectados (FAO, 2020e; FAO, 2020f).

En condiciones normales, en regiones donde existe la transición de las cuatro estaciones, la tendencia general es hacia un consumo de bananos de carácter temporal, pudiéndose observar caídas en el consumo en verano y durante el fin de año, que coincide con aumento de la oferta y demanda de frutas locales de temporada, debido a que las temperaturas puntuales atraen al consumidor hacia otros productos, además de la baja actividad escolar por periodos de vacaciones. El consumo alcanza su máximo en otoño y a principios de la primavera (abril, mayo y octubre), gracias a temperaturas favorables para el consumo del banano (ONU, 2016).

En la última década, se observó que la demanda creció más rápido en los países desarrollados y el consumo per cápita aumentó $2,5 \%$ anual. El banano tiene el mayor consumo dentro del rubro de las frutas y se considera que su valor per cápita es estable a nivel mundial desde 2010 (FAO, 2020a).

Con respecto al comercio, en el contexto actual de contracción económica mundial, el impacto que las exportaciones de LAC puedan tener en los años 2020 y 2021, dependerá en buena medida de la reducción de la demanda externa de productos alimenticios de los principales socios comerciales y de la capacidad de los países para reasignar las exportaciones entre los destinos actuales menos afectados por la crisis de la COVID-19 y para encontrar nuevos socios comerciales relevantes (FAO, 2020d). Las medidas de restricción de movilidad, pueden tener impactos a lo largo de esa cadena de suministro, que incluye afectaciones de los eslabones de la cadena como transporte (internacional o nacional), procesamiento, envasado, almacenamiento, distribución, grandes mercados de abasto y los puntos de venta al detalle, además de tiendas, supermercados, restaurantes y ventas ambulantes (FAO, 2020e).

Un análisis de riesgos y vulnerabilidades en los sistemas alimentarios de los países, puede ayudar a identificar áreas de prioridad y principales riesgos a los que se enfrentan, para desarrollar políticas y programas. El principal riesgo en corto plazo es no poder garantizar el acceso a los alimentos de la población que está cumpliendo con las medidas de seguridad sanitaria para evitar la propagación del virus y, que en muchos casos, han perdido sus fuentes de ingresos por el cese de actividades económicas no esenciales (FAO, 2020e).

Múltiples factores pueden explicar las diferencias entre los países en cuanto a la respuesta de las exportaciones a la crisis actual y no se dispone de datos para poder discutir las causas individuales, las cuales podrían estar basadas en el mercado y/o limitaciones relacionadas con la movilidad laboral durante la pandemia (FAO, 2020e).

La evolución del mercado internacional del banano, guarda estrecha relación con la verticalidad de esta industria y su carácter exportador, que depende de su producción. A través de la historia, se han suscitado cambios en las técnicas de producción del cultivo y canales de comercialización, que determinan su futuro (Martínez et al., 2007) y, ante la situación generada por la pandemia de COVID-19, todo este panorama cambia en forma radical.

En términos generales, el comercio internacional del banano siempre ha estado favorecido por la presencia histórica de empresas trasnacionales (Dole, Chiquita, Fyffes, Del Monte, Compagnie Fruitière, entre otras) que han estructurado y coordinado la oferta en el mercado y que están presentes en casi todos los eslabones de la cadena (producción, transporte, importación, maduración y distribución) (ONU, 2016). No obstante, factores asociados en primer término a la pandemia y luego a otros no menos importantes como el cambio climático, se encuentran entre los principales elementos que afectan en forma directa a la oferta mundial, al incidir sobre las zonas productoras más importantes, lo cual afecta de manera temporal su producción.

En las circunstancias actuales, el mayor impacto estará dirigido hacia los pequeños y medianos productores, mientras que para los grandes exportadores o empresas trasnacionales, debido a sus contratos existentes con los importadores, el impacto es menor. A continuación, se indica el comportamiento de las exportaciones e importaciones, previas al efecto de la pandemia (año 2019), de acuerdo con la Organización de Naciones Unidas para la Alimentación y la Agricultura (FAO, 2020c): 


\section{Exportaciones:}

1) Región asiática: sus exportaciones mostraron recuperación con respecto a la escasez de producción provocada por el clima y brotes de marchitez por Fusarium raza 4 tropical (Foc R4T) entre 2015 y 2017. Sus dos mercados principales han sido China y Japón. En las condiciones actuales, el coronavirus puede limitar el suministro de bananos, se estima una caída promedio de $40 \%$ en las exportaciones provenientes de varias regiones, entre las que desataca Filipinas. Sin embargo, Camboya señala que sus envíos son poco afectados por la pandemia, pero detectan baja en los precios en el mercado internacional, debido a que no se compra la fruta fresca, porque las personas no quieren salir de sus casas (International Tropical Fruits Network, 2020a; 2020b; 2020c) (Figura 2).

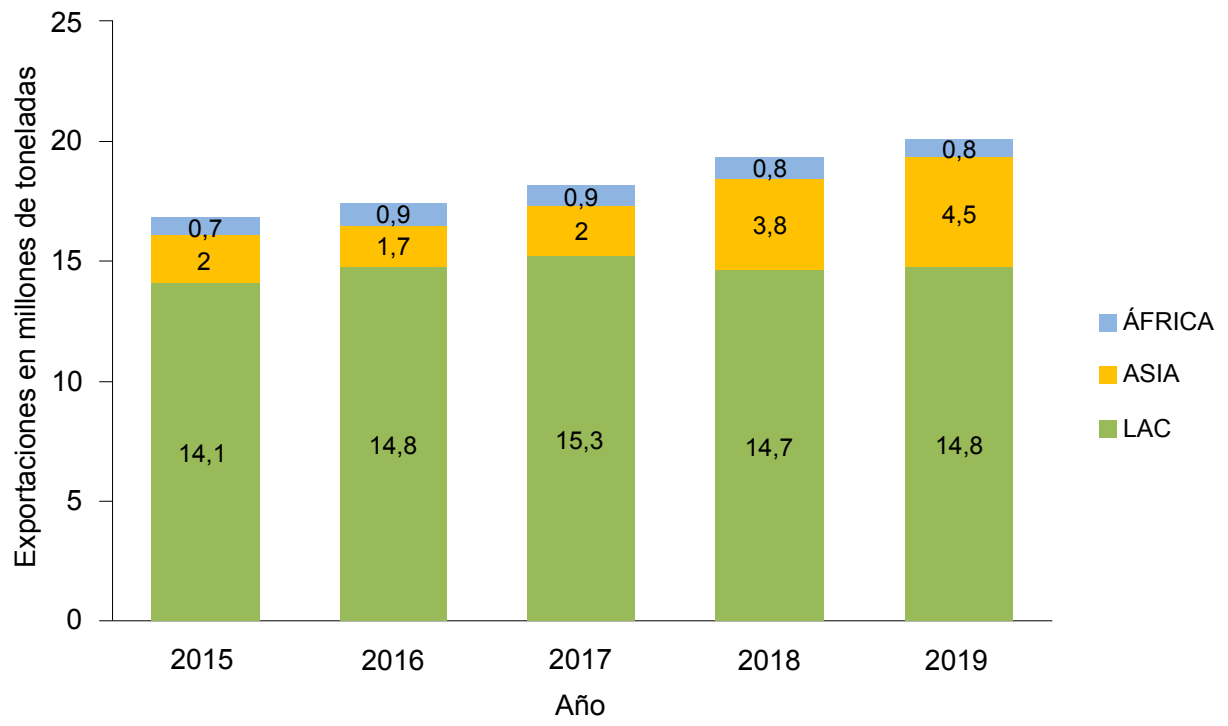

Figura 2. Exportaciones mundiales de banano por región, 2015-2019 (previsión), Fuente: FAO (2020c).

Figure 2. World banana exports by region, 2015-2019 (forecast). Source: FAO (2020c).

2) Región LAC: Ecuador se destaca como el principal exportador, y representaría más del $40 \%$ de las exportaciones de la región, esto a pesar que la mayoría de los países, por efecto de la pandemia, han experimentado un descenso en el total de exportaciones, lo que incluye las frutas. En este país se ha observado un incremento de $0,3 \%$ del total de exportaciones si se comparan los meses entre enero y abril de 2019 y 2020 y un incremento de más de $23 \%$ en el caso de las frutas y en forma principal el banano (FAO, 2020f). Guatemala se presenta como el tercer exportador (después de Ecuador y Filipinas), con aumento en sus exportaciones de $6 \%$. Colombia, logró incremento del $9 \%$ al aumentar su superficie sembrada y aumento de su productividad, para contrarrestar efectos adversos del clima y presencia del Foc R4T. Costa Rica, presentó descenso estimado de $33 \%$, debido a bajas temperaturas, humedad e inundaciones, que benefició las exportaciones de Panamá. Mientras que República Dominicana, a pesar de adversidades del clima, representó el $90 \%$ de las exportaciones del Caribe (Figura 2).

3) Región África: Côte d'Ivoire fue el principal exportador de la región con aumento de 12 \% y Camerún fue el segundo exportador, el cual registró un ligero aumento (0,5\%) (Figura 2). 
Importaciones: los volúmenes de importación mundial ascendieron 18,9 millones de toneladas. Se indica una contracción del 1 \% y del 4 \% en los mayores importadores, Unión Europea y los Estados Unidos, respectivamente. La oferta superior a la demanda siguió disminuyendo los precios en ambos mercados en 2019, durante los meses de verano, por la competencia con las frutas de zonas templadas (FAO, 2020c).

La Unión Europea, mayor importador mundial, presentó disminución ligera del volumen importado, al igual que los precios de importación que fueron $2 \%$ más bajos (FAO, 2020c).

Estados Unidos se presentó como el segundo mayor importador, con una disminución del volumen mundial estimada del $22 \%$. Al igual que la Unión Europea, se debió a la competencia por las frutas de verano.

China ha presentado una expansión estimada del $36 \%$ a partir de 2018. La demanda siguió impulsada por el rápido crecimiento de los ingresos y los cambios conexos en las preferencias de los consumidores. Su cuota se incrementó en un $12 \%$ de las importaciones netas mundiales y superó a Rusia (tercer mayor importador en el mundo). Sus importaciones se incrementaron $125 \%$, las cuales procedieron de Ecuador, las cuales duplicaron el volumen proveniente de Vietnam y mantuvo a Filipinas como proveedor habitual (FAO, 2020c).

Rusia presentó una disminución del $4 \%$, tras haber logrado un considerable aumento en 2016 y 2017, gracias al fortalecimiento de la moneda, el aumento de los ingresos y los cambios en las preferencias de los consumidores. Mientras que Japón presentó un crecimiento moderado del $2 \%$ en 2019, debido a la creciente popularidad del banano entre los consumidores. El crecimiento de sus importaciones se benefició de la recuperación de la producción en Filipinas, el mayor proveedor (FAO, 2020c).

\section{Amenazas fitosanitarias vigentes}

La producción de musáceas comestibles a través de diversos modelos de producción (desde cultivos traspatio hasta sofisticados sistemas de plantación), dependen en esencia de cambios en los parámetros que componen el sistema clima, expresados a través del concepto de cambio climático. Una consecuencia directa del mismo, es el incremento de incidencias de plagas y patógenos, además, al considerar la existencia de una base genética uniforme, estas amenazas fitosanitarias son consideradas como las principales limitaciones responsables de las pérdidas de rendimiento y baja productividad (Higuera, 2015).

En términos generales, se prevé que en las regiones con incremento de lluvias y humedad relativa, se aumenten las enfermedades como Sigatoka negra y fusariosis, mientras que en aquellas con tendencia a sequía, las poblaciones de áfidos (vectores virales) y trips (Higuera, 2015). De igual manera, otras limitaciones fitosanitarias como Moko (Ralstonia solanacearum) y pudricion bacterial por Erwinia (Erwinia sp.), pueden aumentar su nivel de agresividad, dado los cambios o alteraciones de las condiciones climáticas (Dita et al., 2020; Martínez-Solórzano et al., 2020). También existen otras amenazas potenciales o emergentes, que deben ser consideradas como peligro latente y que se indican a continuación:

Marchitez por Fusarium Raza 4 (Foc R4T): es causada por el hongo Fusarium oxysporum f. sp. cubense, raza 4, variantes tropical y subtropical y es considerada entre las diez enfermedades vegetales más importantes en la historia de la agricultura; al no existir control, puede perpetuarse en el suelo durante largo periodo y no existen clones resistentes. En la actualidad, bananos del subgrupo Cavendish (clones susceptibles a esta raza), cubren la mayor área global y el mercado internacional (casi en su totalidad), por cuanto la evidente expansión geográfica de la Foc RT4, con su potencial efecto perjudicial, se conjugan sobre la premisa de que los factores que contribuyeron con la expansión de la R1, están vigentes para su diseminación (Dita et al., 2020; Martínez et al., 2020).

Las proyecciones sugieren que una propagación ulterior de Foc R4T, significaría enormes pérdidas de ingresos y empleos en países afectados. Al observar el posible impacto sobre la producción bananera por región, las pérdidas agregadas serían más pronunciadas en Asia, afectarán el mercado mundial y generarán un aumento en el precio 
de referencia mundial, por cuanto los productores de los países no afectados estarían estimulados a aumentar su producción (FAO, 2019). Dada la condición que este hongo puede sobrevivir en el suelo por más de veinte años, conlleva a que las unidades de producción infectadas no pueden ser utilizadas para muchos clones susceptibles a esta raza durante decenios, lo que implica el desplazamiento de su producción a nuevas tierras no afectadas. Por consiguiente, y de acuerdo con la gravedad de la propagación, la alta velocidad con que aparecen nuevos brotes de la enfermedad, puede provocar una escasez cada vez mayor de suelos libres del patógeno (FAO, 2019).

El Foc R4T representa una amenaza potencial para los pequeños productores de regiones afectadas, que carecen de medios necesarios para mantener sus operaciones frente a las pérdidas de rendimiento y el aumento de los costos de producción. La prevención, la contención rápida y la cuarentena son importantes. En julio de 2019, el hongo fue detectado por primera vez en plantaciones de banano en América Latina, en la región nororiental de La Guajira, Colombia, lo que activó la alarma en toda la región de América Latina y el Caribe, al ser el primer reporte de la enfermedad en el continente americano (FAO, 2019; Martínez et al., 2020). Posteriormente en abril del año 2021 fue reportado en Piura, Perú, zona cercana a la frontera con Ecuador (principal productor mundial) (Servicio Nacional de Sanidad Agraria del Perú, 2021).

Bunchy Top Virus (BBTV): es una enfermedad viral catalogada como la más importante, que amenaza latentemente el género Musa a nivel mundial, y fue nominada entre las cien peores. Es el principal problema que afecta muchas áreas del Sureste de Asia, Pacífico y, en menor medida, en el continente africano, puede generar pérdidas de hasta del $100 \%$ (Qazi, 2016). No hay registros de mismo en el Nuevo Mundo, excepto en Hawái (Blomme et al., 2013; Qazi, 2016). Es transmitido en forma local por Pentalonia nigronervosa, a larga distancia a través de material vegetal infectado (retoños y cormos) utilizados para la siembra. No se transmite mecánica o por contacto con implementos agrícolas (Qazi, 2016). El uso de plantas in vitro no solo es la mayor fuente de material de siembra libre de virus, sino que también es una excelente estrategia de control, debido que una vez establecida, es muy difícil controlar o erradicar. Plantas transgénicas con resistencia a BBTV han sido probadas en Australia, Hawái e India, con base en el enfoque de resistencia derivada de patógenos. (Qazi, 2016).

Marchitamiento por xanthomonas (Xanthomonas campestris pv. Musacearum): esta bacteria puede atacar todos los cultivares de Musa, puede generar pérdidas de rendimiento de hasta $100 \%$, en clones tipo ABB. Compromete la seguridad alimentaria y medios de vida de hogares de agricultores a base de banano. Los síntomas se observaron por primera vez en Ensete en Etiopía en 1930 y, desde el 2001, se ha extendido desde Uganda, este de la República Democrática del Congo, Ruanda, Tanzania, Kenia y Burundi (Blomme et al., 2017).

Enfermedad sangrado del banano (Ralstonia syzygii Subsp. Celebesensis): se originó en la isla Salayar, cerca de Sulawesi, donde estuvo confinada durante muchos años por las estrictas regulaciones de cuarentena. Sin embargo, se había generalizado en los cultivares locales en 1920 y luego se extendió por toda la isla hasta su reporte en Java a fines de la década de 1980. El patógeno ha continuado su propagación a la mayoría de las islas más grandes de Indonesia, donde las pérdidas de rendimiento promedio van en aumento (Blomme et al., 2017). Estos brotes se asociaron con la transmigración de personas de Java a islas menos pobladas de Indonesia. La enfermedad se está extendiendo en Malasia peninsular, donde coexiste con las enfermedades de marchitez de Moko y Fusarium, de igual manera en Nueva Guinea y en Java Occidental, donde se registró pérdida de plantaciones (Blomme et al., 2017).

\section{Conclusiones}

Por sus cualidades y aporte nutritivo (vitaminas, minerales y otros elementos con propiedades medicinales), los bananos son considerados como las frutas más consumidas y cultivos estratégicos en la seguridad alimentaria de muchos países, destacándose entre los demás por su rápido crecimiento en la agricultura, a pesar de la existencia de muchas incertidumbres causadas por diversas enfermedades, perturbaciones climáticas, entre otras, que pueden 
afectar los entornos económicos potencialmente volátiles y su desarrollo económico. En la actualidad, su producción y comercio, tienen como reto adaptarse y responder ante situaciones generadas por dos pandemias: el coronavirus y la marchitez por Fusarium Foc R4T, las cuales se han expandido a nivel global a pesar de implementarse medidas de bioseguridad que incluyen cuarentena y aislamiento de áreas para mitigar su propagación.

En el caso de América Latina y el Caribe, esta región se encuentra a la expectativa ante la reciente llegada de Foc R4T y la COVID-19, que originará un punto de inflexión donde el principal desafío es mantener los niveles de producción para cumplir con la distribución y abastecimiento entre la población, ante estas inminentes amenazas.

\section{Referencias}

Anyasi, T. A., Jideani, A., \& Mchau, G. (2013). Functional properties and post-harvest utilization of commercial and noncommercial banana cultivars. Comprehensive Reviews in Food Science and Food Safety, 12(5), 509-522. https:// doi.org/10.1111/1541-4337.12025

Arawande, J., \& Komolafe, E. (2010). Antioxidative potentials of banana and plantain peel extracts on crude palm oil. Ethnobotanical Leaflets, 14, 559-569. http://www.ethnoleaflets.com/

Blasco, G., \& Gómez, F. (2014). Propiedades funcionales del banano (Musa sp.). Revista Médica de la Universidad Veracruzana, $14(2), 22-26$.

Blomme, G., Dita, M., Jacobsen, K. S., Pérez Vicente, L., Molina, A., Ocimati, W., Poussier, S., \& Prior, P. (2017). Bacterial diseases of bananas and enset: current state of knowledge and integrated approaches toward sustainable management. Frontiers in Plant Science, 8, Article 1290. https://doi.org/10.3389/fpls.2017.01290

Blomme, G., Ploetz, R., Jones, D., Langhe, E. Price, N., Gold, C., Geering, A, Viljoen, A., Karamura, D., Pillay, M., Tinzaara, W., Teycheney, P., Lepoint, P., Karamura, E., \& Buddenhagen, I. (2013). A historical overview of the appearance and spread of Musa pests and pathogens on the African continent: Highlighting the importance of clean Musa planting materials and quarantine measures. Annals of Applied Biology, 162(1), 4-26. https://doi.org/10.1111/aab.12002

China, R., Dutta, S., \& Sen, S. (2011). In vitro antioxidant activity of different cultivars of banana flower (Musa paradicicus L.) extracts available in India. Journal of Food Science, 76(9), C1292-C1299. https://doi.org/10.1111/j.17503841.2011.02395.x

Crawford, A., \& Kueffner, S. (2020). Disease Is Ravaging the \$25 Billion Banana Industry. Bloomberg. https://www.bloomberg. $\mathrm{com} /$ news/features/2020-05-22/the-25-billion-banana-industry-is-being-ravaged-by-disease?srnd=premiumasia\&sref=Mkhc1AWW

Dita, M., Teixeira, L. A. J., O’Neill, W., Pattison, A. B., Weinert, M. P., Li, C. Y., Zheng, S. J., Staver, C., Thangavelu, R., \& Viljoen, A. (2020). Current state of Fusarium wilt of banana in the subtropics. Acta Horticulturae, 1272, 45-56. https://doi.org/10.17660/ActaHortic.2020.1272.7

Emaga, T., Andrianaivo, R., Whatelet, B., Techango, J., \& Paquot, M. (2007). Effects of the stage of maturation and varieties on the chemical composition of banana and plantain peels. Food Chemistry, 103(2), 590-600. https://doi.org/10.1016/j. foodchem.2006.09.006Ge

Food and Agriculture Organization. (2020a). Perspectivas a mediano plazo: perspectivas para la producción y el comercio mundial de bananos y frutas tropicales 2019-2028. http:/www.fao.org/economic/est/est-commodities/banano/es/

Food and Agriculture Organization. (2020b). Base datos: Superficie, producción y exportación de banano y plátano. FAOSTAT. http://www.fao.org/faostat/es/\#home (consultado 05 mar. 2020). 
Food and Agriculture Organization. (2020c). Análisis del mercado del banano: resultados preliminares 2019. http://www.fao. org/economic/est/est-commodities/banano/es/

Food and Agriculture Organization. (2020d). COVID-19: destino y diversificación de las exportaciones de alimentos en América Latina y el Caribe. FAO. https://doi.org/10.4060/CA9267ES

Food and Agriculture Organization. (2020e). Seguridad Alimentaria bajo la Pandemia de COVID-19. http://www.fao.org/3/ ca8873es/CA8873ES.pdf

Food and Agriculture Organization. (2020f). COVID-19: Crecimiento de las exportaciones de frutas y verduras en comparación con el crecimiento total de las exportaciones. FAO. https://doi.org/10.4060/CA9270ES

Food and Agriculture Organization. (2019). La marchitez del banano por Fusarium Raza 4 Tropical: ¿Una creciente amenaza al mercado mundial del banano? En "Enfoque de productos básicos. Perspectivas Alimentarias de FAO. http://www. fao.org/3/ca6911en/ca6911en.pdf

Gavrovic-Jankulovic, M., \& Petersen, A. (2008). A novel recombinantly produced banana lectin isoform is a valuable tool for glycoproteomics and a potent modulator of the proliferation response in $\mathrm{CD}^{+}, \mathrm{CD} 4^{+}$, and CD8+ populations of human PBMCs. The International Journal of Biochemistry \& Cell Biology, 40(5), 929-941. https://doi.org/10.1016/j. biocel.2007.10.033

Hapsari, L., \& Lestari, D. (2016). Fruit characteristic and nutrient values of four Indonesian banana cultivars (Musa spp.) at different genomic groups. AGRIVITA Journal of Agricultural Science, 38(3), 303-311. http://dx.doi.org/10.17503/ agrivita.v38i3.696

Hernández, L., \& Vit., P. (2009). El plátano: Un cultivo tradicional con importancia nutricional. Revista Colegio de Farmacéuticos del Estado Mérida, 2(13), 11-14. https://www.academia.edu/32367278/El_pl\%C3\%A1tano_un_ cultivo_tradicional_con_importancia_nutricional

Heslop-Harrison, J., \& Schwarzacher, T. (2007). Domestication, genomics and the future for banana. Annals of Botany, 100, 1073-1084. https://doi.org/10.1093/aob/mcm191

Higuera C. (2015). Bananos y plátanos, frente al cambio climático. En I. Higuera (Ed.), Hacia dónde va la ciencia en México (pp. 61-81). CONACYT,Academia Mexicana de Ciencias A.C., \& Consejo Consultivo de Ciencias. https://www.researchgate. net/publication/292985144_BANANOS_Y_PLATANOS_FRENTE_AL_CAMBIO_CLIMATICO/citation/download

International Tropical Fruits Network. (2020a). CAMBODIA: Banana exports solid but mangoes struggling as Novel Coronavirus affects fresh fruit markets. https://www.itfnet.org/v1/2020/02/cambodia-banana-exports-solid-butmangoes-struggling-as-novel-coronavirus-affects-fresh-fruit-markets/ 1/5

International Tropical Fruits Network. (2020b). PHILIPPINES: Banana group says coronavirus dampens exports to China. https://www.itfnet.org/v1/2020/02/philippines-banana-group-says-coronavirus-dampens-exports-to-china/ 1/4

International Tropical Fruits Network. (2020c). USA: Coronavirus is about to hit banana supplies next. https://www.itfnet.org/ v1/2020/04/usa-coronavirus-is-about-to-hit-banana-supplies-next/ 1/5

Kanazawa, K., \& Sakakibara, H. (2000). High content of dopamine, a strong antioxidant, in Cavendish banana. Journal of Agricultural and Food Chemistry, 48, 844-848. https://doi.org/10.1021/jf9909860

Kumar, K., Bhowmik, D., Duraivel, S., \& Umadevi, M. (2012). Traditional and Medicinal Uses of Banana. Journal of Pharmacognosy and Phytochemistry, 1(3), 51-63. https://www.researchgate.net/publication/285484754_Traditional_ and_medicinal_uses_of_banana

Li, L., Wang, H., Zhang, C., Wang, X., \& Shi, F. (2013). Origins and Domestication of Cultivated Banana Inferred from Chloroplast and Nuclear Genes. PLoS ONE, 8(11), Article e80502. https://doi.org/10.1371/journal.pone.0080502 
Martínez, G., Delgado, E., Pargas, R., Manzanilla, E., \& Ramírez, H. (2007). Consideraciones generales sobre la producción y el comercio mundial de banano. I: Producción, exportación e importación. CENIAP HOY No 13 . https:// www.researchgate.net/publication/298807693_Consideraciones_generales_sobre_la_produccion_y_el_comercio_ mundial_de_banano_I_Produccion_exportacion_e_importacion/citation/download

Martínez-Solórzano, G., Rey-Brina, J., Pargas-Pichardo, R., \& Manzanilla, E. (2020). Marchitez por Fusarium raza tropical 4: Estado actual y presencia en el continente americano. Revista Agronomía Mesoamericana, 31(1), 259-276. https:// doi.org/10.15517/am.v31i1.37925

Organización de las Naciones Unidas. (2016). BANANO. Conferencia de las Naciones Unidas sobre comercio y desarrollo. https://unctad.org/es/system/files/official-document/INFOCOMM_cp01_Banana_es.pdf

Perrier X., Langhe E. D., Donohue, M., Lentfer, C., \& Vrydaghs, L. (2011). Multidisciplinary perspectives on banana (Musa spp.) domestication. Proceeding of National Academy of Sciences of the United States of America, 108(28), 1131111318. https://doi.org/10.1073/pnas.1102001108

Qazi, J. (2016). Banana bunchy top virus and the bunchy top disease. Journal of General Plant Pathology, 82(1), 2-11. https:// doi.org/10.1007/s10327-015-0642-7

Rekha A., Ravishankar K., Anand, L., \& Hiremath S. (2001). Diversidad genética y genómica en banano (especies y cultivares de musa) basada en el análisis D2 y marcadores RAPD. INFOMUSA, 10(2), 29-34.

Saif, M., \& Hashinada, F. (2005). Antibacterial and antioxidant activities of banana (Musa, AAA cv. Cavendish) fruits peel. American Journal of Biochemistry and Biotechnology, 1(3), 125-131. https://doi.org/10.3844/ajbbsp.2005.125.131

Saravanan, K., \& Aradhya, S. (2011). Potential nutraceutical food beverage with antioxidant properties from banana plant biowaste (pseudostem and rhizome). Food \& Function, 2(10), 603-610. https://doi.org/10.1039/c1fo10071h

Servicio Nacional de Sanidad Agraria del Perú. 2021. SENASA confirma brote de Fusarium raza 4 tropical en Piura. https:// www.gob.pe/institucion/senasa/noticias/429832-senasa-confirma-brote-de-fusarium-raza-4-tropical-en-piura

Someya, S., Yoshiki, Y., \& Okubo, K. (2002). Antioxidant compounds from bananas (Musa Cavendish). Food Chemistry, 79(3), 351-54. http://dx.doi.org/10.1016/S0308-8146(02)00186-3

Soto, M. (1992). Banano cultivo y comercialización (2 ${ }^{\mathrm{da}}$ Ed). Litografía e Imprenta LIL.

Swanson M., \& Markovitz, D. (2015). Engineering a therapeutic lectin by uncoupling mitogenicity from antiviral activity. Cell, 163(3), 746-758. https://doi.org/10.1016/j.cell.2015.09.056

Ugye,T., \& Nyiaatagher,T.(2009). Chemical composition of Musa Sapientum (Banana) peels.Electronic Journal of Environmental, Agricultural and Food Chemistry, 8(6), 437-442. https://www.researchgate.net/publication/233760453_Chemical_ Composition_of_Musa_sepientum_Banana_Peels

Wachirasiri, P., Julakarangka S., \& Wanlapa, S. (2009). The effects of banana peel preparations on the properties of banana peel dietary fiber concentrate. Journal Science and Technology, 31(6), 605-611. https://www.researchgate.net/ publication/41162819_The_effects_of_banana_peel_preparations_on_the_properties_of_banana_peel_dietary_fibre_ concentrate/citation/download

Zomo, S. A., Ismail, S. M., Jahan, M. S., Kabir, K., \& Kabir, M. H. (2014). Chemical properties and shelf life of banana (Musa sapientum L.) as influenced by different postharvest treatments. The Agriculturists, 12(2), 6-17. https://doi. org/10.3329/agric.v12i2.21725 